Document downloaded from:

http://hdl.handle.net/10251/141525

This paper must be cited as:

Ten-Domenech. I.; Martínez-Pérez-Cejuela, H.; Simó-Alfonso, E.; Torres-Cartas, S.; Meseguer-Lloret, S.; Herrero Martínez, J. (2018). Polymer-based materials modified with magnetite nanoparticles for enrichment of phospholipids. Talanta. 180:162-167. https://doi.org/10.1016/j.talanta.2017.12.042

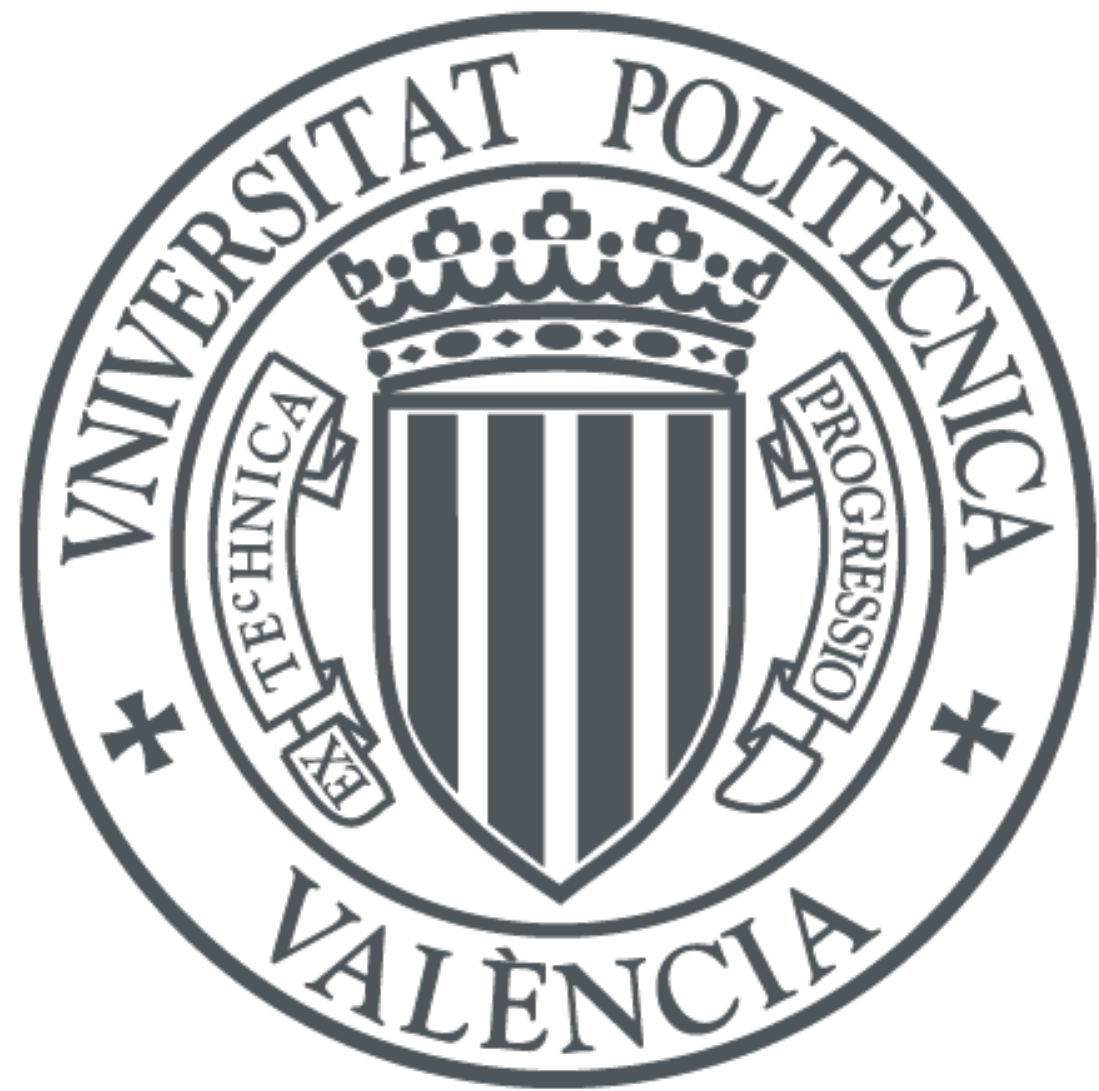

The final publication is available at

https://doi.org/10.1016/j.talanta.2017.12.042

Copyright Elsevier

Additional Information 


\title{
Polymer-based materials modified with magnetite nanoparticles for enrichment of phospholipids
}

\author{
I. Ten-Doménech ${ }^{\text {a }}$ H. Martínez-Pérez-Cejuela ${ }^{a}$, E. F. Simó-Alfonso a , S. Torres- \\ $\operatorname{Cartas}^{\mathrm{b}}$, S. Meseguer-Lloret ${ }^{\mathrm{b}}$, J. M. Herrero-Martínez ${ }^{\mathrm{a},{ }^{*}}$ \\ ${ }^{a}$ Department of Analytical Chemistry, University of Valencia, C/Doctor Moliner 50, 46100 \\ Burjassot, Valencia \\ ${ }^{b}$ Instituto de Investigación para la Gestión Integrada de Zonas Costeras, Universitat \\ Politècnica de València, C/Paraninf 1, 46730 Grao de Gandía, Spain \\ * Corresponding author: Dr. José Manuel Herrero Martínez ( jmherrer@uv.es)
}

\begin{abstract}
A polymeric material modified with magnetic nanoparticles (MNPs) has been synthesized and evaluated as sorbent both for solid-phase extraction (SPE) and dispersive magnetic solid-phase extraction (MSPE) of phospholipids (PLs) in human milk samples. The synthesized sorbent was characterized by scanning electron microscopy and its iron content was also determined. Several experimental variables that affect the extraction performance (e.g. loading solvent, breakthrough volume and loading capacity) were investigated and a comparison between conventional SPE and MSPE modalities was done. The proposed method was satisfactorily applied to the analysis of PLs in human milk fat extracts in different lactation stages and the extracted PLs were determined by means of hydrophilic interaction liquid chromatography using evaporative light scattering detection.
\end{abstract}

Keywords: magnetic polymer-based material; solid-phase extraction; phospholipids; human milk; hydrophilic interaction liquid chromatography-evaporative light scattering detection 


\section{Introduction}

Human breast milk can be considered as the optimum food for all infants, since it satisfies the nutritional requirements for a healthy growth and development. Among the different human milk nutrients, about $50 \%$ of total energy intake comes from the lipid fraction of milk [1]. Within this fraction, phospholipids (PLs) are basic constituents of the milk fat globule membrane, showing emulsifying properties and, moreover, their implication in many biological processes (e.g. brain development in newborn infants) has been demonstrated [2]. The total amount of PLs in human milk ranges from 0.5 to $1.0 \mathrm{~g}$ per $100 \mathrm{~g}$ of fat [3] and their distribution, given as percentage of total PLs, is: phosphatidylcholine (PC) 28-35\%, phosphatidylethanolamine (PE) 28-30\%, sphingomyelin (SM) 25-38\%, phosphatidylinositol 59\% and phosphatidylserine 3-9\% [4,5].

Due to the amphiphilic nature of PLs, their quantitative analysis is not a straightforward process. To characterize and quantify PLs in both biological and food matrices, hydrophilic interaction liquid chromatography (HILIC), either coupled with evaporative light scattering detection (ELSD) [6] or with mass spectrometry (MS) [7], has been satisfactorily applied .

However, the challenge in PLs analysis arises from their low abundance with respect to nonpolar triglycerides (98-99\%). To address this problem, several techniques, such as twodimensional thin-layer chromatography [8,9], column chromatography [10] and solid-phase extraction (SPE) [5-7] have been employed.

In this context, magnetic materials have recently emerged as extraction sorbents (namely, magnetic solid-phase extraction (MSPE)) in the analysis of food samples and other matrices [11,12]. These sorbents can be easily handled by the application of an external magnetic field (a magnet arranged outside the extraction vessel), thereby avoiding additional centrifugation or filtration steps. A usual strategy to synthesize functionalized magnetic nanomaterials (FMMs) is to coat the core of magnetic nanoparticles (MNPs) with silica via solgel process [13]. Alternatively, polymer-coated MNPs can be accomplished by physical/chemical adsorption or surface coating with specific ligands, depending on specific applications. However, some of these FMMs showed limited dispersibility and reproducibility, which undoubtedly affect to their adsorption performance. In this sense, the immobilization of bare MNPs onto silica or polymeric supports could be an alternative approach to be considered. This procedure would allow to fully exploit their interaction capabilities, as for instance, with phosphorus-based compounds $[14,15]$.

Recently, organic polymers have been synthesized by in situ preparation and evaluated as alternative stationary phases to replace conventional packing materials [16]. These materials show some advantages, such as their high stability, low cost, ease of preparation and very low resistance to flow. Particularly, polymeric monoliths based on glycidyl methacrylate (GMA) have been recently employed as platform in the synthesis of novel sorbents with different 
functionalities for sample preparation purposes [16-18]. However, the use of GMA as support for the attachment of MNPs has been only slightly explored $[19,20]$. Although these supports have been applied to the enrichment of phosphorous-compounds in aqueous samples (with a reduced number of possible interferences), its application to the isolation of phospholipids in more complex matrices (lipid matrices) has not been explored.

The aim of this work was the evaluation of a composite material, which combines the advantages of MNPs and GMA polymers to selectively extract PLs from real complex matrices such as human milk. The sorbent was prepared from a ground GMA-co-ethylene glycol dimethacrylate (EDMA) polymer, subsequently modified with a silanizing agent, to which MNPs were attached. This procedure provides a sorbent with a surface coated with bare MNPs, which are available to interact with the phosphate groups present in PLs. The prepared material was evaluated both in conventional SPE and in MSPE to extract PC from standard solutions. The features of this material as extraction sorbent (such as extraction recoveries, reusability, etc) were studied. After conventional SPE or MSPE treatment, HILIC-ELSD analysis was carried out. To our knowledge, this is the first work dealing with PLs isolation from human milk fat extracts using a polymeric sorbent modified with MNPs.

\section{Materials and methods}

\subsection{Chemicals and reagents}

HPLC-grade acetonitrile $(\mathrm{ACN})$, methanol $(\mathrm{MeOH})$ and chloroform were purchased from VWR Chemicals (Barcelona, Spain); reagent-grade dichloromethane, ethanol (EtOH), $n$ hexane, sulfuric acid and hydrochloric acid, anhydrous sodium sulfate, sodium hydroxide, ascorbic acid and ammonia were supplied by Scharlab (Barcelona, Spain); glycidyl methacrylate (GMA), ethylene dimethacrylate (EDMA), o-phenanthroline monohydrate, sphingomyelin (chicken egg yolk, $\geq 95 \%$ ) (SM) and L- $\alpha$-phosphatidylethanolamine (egg yolk, $\geq 97 \%$ ) (PE) were obtained from Sigma-Aldrich (St. Louis, MO, USA); azobisisobutyronitrile (AIBN), butylated hydroxytoluene and ammonium formate were supplied by Fluka (Buchs SG, Switzerland); cyclohexanol, 1-dodecanol, (3-aminopropyl)trimethoxysilane (APTMS), 2nitrophenol and acetone were purchased from Alfa-Aesar (Karlsruhe, Germany); ferrous chloride $\left(\mathrm{FeCl}_{2} \cdot 4 \mathrm{H}_{2} \mathrm{O}\right)$, ferric chloride $\left(\mathrm{FeCl}_{3} \cdot 6 \mathrm{H}_{2} \mathrm{O}\right)$, citric acid monohydrate and ammonium thiocyanate were supplied by Panreac (Barcelona, Spain); ammonium iron (II) sulfate hexahydrate (Mohr's salt) was obtained from Probus (Badalona, Spain); L- $\alpha$ phosphatidylcholine (soy bean, 95\%) (PC) was purchased from Avanti Polar Lipids (Alabaster, AL, USA). 
Deionized water (Barnstead deionizer, Sybron, Boston, Mass., U.S.A.) was used in all procedures.

Empty propylene cartridges ( $3 \mathrm{~mL})$ and frits ( $20 \mu \mathrm{m}$ pore size) were supplied by Análisis Vínicos (Tomelloso, Spain).

\subsection{Instrumentation}

Polymerization protocol was carried out using a vacuum oven (Memmert, Schwabach, Germany).

Transmission electron microscope (TEM) images of the MNPs were obtained using a Jeol (Tokyo, Japan) model JEM-1010 microscope operated at $100 \mathrm{kV}$. Images were obtained using a MegaView III camera provided with the AnalySIS image data acquisition system.

Scanning electron microscope (SEM) images were obtained with a Hitachi S-4800 (Ibaraki, Japan) integrated with backscattered electron detector (Bruker, Germany).

Surface areas were measured by porosimetry using nitrogen adsorption-desorption isotherms. The isotherms were recorded with a Micromeritics ASAP2020 automated sorption analyzer. The specific surface areas were calculated from the adsorption data in low-pressure range using BET model.

Inductively-coupled plasma MS (ICP-MS) measurements were conducted with an Agilent 7900 ICP-MS (Agilent Technologies, Waldbronn, Germany).

For UV-Vis measurements, an 8453 diode-array UV-Vis spectrophotometer (Agilent Technologies) was used.

Centrifugation steps were conducted in a Hettich ${ }^{\circledR}$ EBA 21 laboratory centrifuge with rotor 1116 and adapters 1631 (Sigma, Osterode am Harz, Germany).

Evaporation under vacuum was accomplished using a miVac sample concentrator (SP Scientific, Warminster, PA).

Chromatographic separation and determination of PLs was made with an 1100 series liquid chromatograph (Agilent Technologies) provided with a quaternary pump, a degasser, a thermostated column compartment, an automatic sampler, a UV-Vis diode array detector online coupled to an Agilent 385-evaporative light scattering detector (ELSD) (see Supporting Information for chromatographic and detection conditions).

\subsection{Preparation of $M N P S$}

$\mathrm{Fe}_{3} \mathrm{O}_{4}$ nanoparticles were synthesized through the co-precipitation method from aqueous $\mathrm{Fe}^{3+} / \mathrm{Fe}^{2+}$ salt solutions with a ratio $2: 1$ by the addition of ammonia [13,21] (see Supporting Information).

\subsection{Synthesis and silanization of GMA-co-EDMA material}


The synthesis of GMA-co-EDMA polymeric material was based on a previous work [22]. The obtained polymeric material was ground with a mortar and sieved to particle sizes between 125 and $200 \mu \mathrm{m}$.

For the silanization, $3 \mathrm{~g}$ of the powdered material were placed in a glass flask with 75 $\mathrm{mL}$ of a $65 \mathrm{mM}$ APTMS solution in acetone and allowed to react for $3 \mathrm{~h}$ at $60^{\circ} \mathrm{C}$ (water bath) under stirring. Then, the silanized material was filtered, washed with $\mathrm{EtOH}$, dried at $60^{\circ} \mathrm{C}$ and stored in a desiccator until use.

\subsection{Functionalization of silanized GMA-based material with MNPs}

The attachment of the MNPs was carried out following the method described by Meseguer-Lloret and co-workers [20]. Thus, the silanized powdered material (2.5 g) was placed into a doubled PTFE fritted $60 \mathrm{~mL}$ propylene syringe. Several fractions $(15 \times 5 \mathrm{~mL})$ of a 1000 $\mu \mathrm{gL}^{-1}$ dispersion of MNPs in EtOH-water $(1: 1, \mathrm{v} / \mathrm{v})$ were passed through the syringe under continuous stirring, while remaining liquid was removed by gravity at a flow rate of $0.5 \mathrm{~mL}$ $\min ^{-1}$. After that, a brown colored powder, corresponding to the MNPs-modified material, was obtained. To remove the excess of MNPs, the material was washed with $100 \mathrm{~mL}$ of an EtOHwater solution $(1: 1, \mathrm{v} / \mathrm{v})$, followed by $15 \mathrm{~mL}$ of EtOH. Then, the MNPs-modified material was dried in an oven at $60^{\circ} \mathrm{C}$ and stored in a desiccator until use. The synthesis route to obtain the MNPs-modified material is shown in Fig. 1.

Fe content of the MNPs-modified material was evaluated in triplicate using both UVVis [23] and ICP-MS (see Supporting Information).

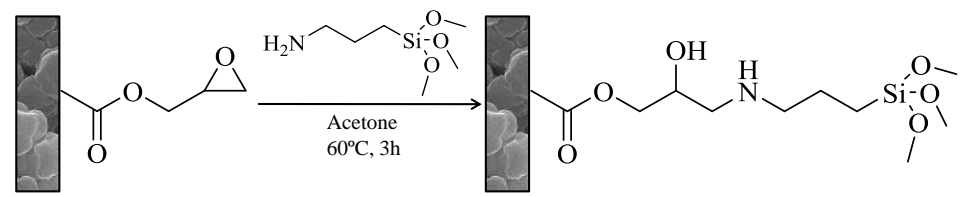

GMA-co-EDMA material Silanized GMA-co-EDMA material

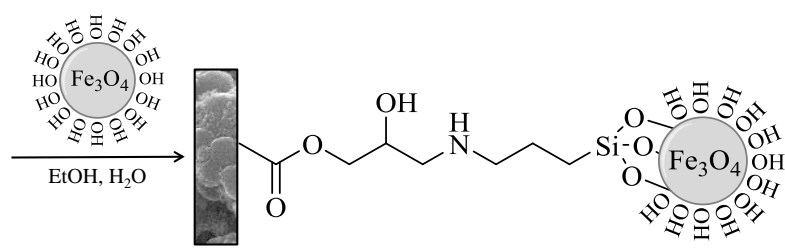

MNPs-modified material

Fig. 1. Scheme of modification with APTMS of the poly(GMA-co-EDMA) material and subsequent functionalization with MNPs. 


\subsection{SPE protocol}

For the preparation of the SPE cartridges, $150 \mathrm{mg}$ of the MNPs-modified material was packed between two frits into $3 \mathrm{~mL}$ empty propylene cartridges. To compact the material, the sorbent was washed under vacuum with $15 \mathrm{~mL}$ of an EtOH-water solution $(1: 1, \mathrm{v} / \mathrm{v})(\mathrm{Fig}$. S2A). Activation and equilibration of the sorbent was done with $\mathrm{MeOH}$ and hexane-EtOH (98:2, v/v), respectively. Then, $500 \mu \mathrm{L}$ of a PC solution of $500 \mu \mathrm{g} \mathrm{mL}^{-1}$ prepared in this later solvent were percolated through the SPE cartridge. Before eluting retained $\mathrm{PC}$ with $\mathrm{CHCl}_{3}: \mathrm{MeOH}(2: 1, \mathrm{v} / \mathrm{v})$ $(500 \mu \mathrm{L})$, a washing step with hexane $(500 \mu \mathrm{L})$ was done. Throughout the SPE process, PC in the loading, washing and elution fractions was quantified by Stewart assay [24]. Then, the sorbent was regenerated with $\mathrm{CHCl}_{3}$ followed by $\mathrm{MeOH}$. The same procedure was also applied to the GMA-co-EDMA polymer (blank).

\subsection{MSPE protocol}

To carry out the MSPE, $150 \mathrm{mg}$ of MNPs was placed in a glass tube and were conditioned with $\mathrm{MeOH}$ followed by hexane-EtOH (98:2, v/v). To collect MNPs in all extraction steps, a neodymium magnet was placed on the wall of the glass tube until no turbidity in the supernatant was observed (Fig. S2B). Then, $5 \mathrm{~mL}$ of a PC standard solution $\left(50 \mu \mathrm{g} \mathrm{mL}^{-1}\right)$ were added and the mixture was vortexed for $1 \mathrm{~min}$. Using the magnet, the supernatant solution was discarded, and the sorbent was washed with $5 \mathrm{~mL}$ of hexane. Next, retained PC was desorbed with $5 \mathrm{~mL} \quad \mathrm{CHCl}_{3}: \mathrm{MeOH} \quad(2: 1, \mathrm{v} / \mathrm{v})$. PC content in each fraction was spectrophotometrically quantified at $488 \mathrm{~nm}$ [24].

\subsection{Fat extraction and analysis of human milk}

In order to extract lipids from human milk samples, the extraction method described by Folch et al. [25] with minor modifications was applied (see Supporting Information). The fat extract was re-dissolved in $5 \mathrm{~mL}$ hexane-EtOH $(98: 2, \mathrm{v} / \mathrm{v})$ and percolated through the cartridge following the protocol described in Section 2.6. The eluted fraction was dried under vacuum, redissolved in a known volume of $\mathrm{CHCl}_{3}: \mathrm{MeOH}(2: 1, \mathrm{v} / \mathrm{v})(250-500 \mu \mathrm{L})$ and injected in the HPLC system.

\section{Results and discussion}

\subsection{Characterization of the MNPs-modified material}

As described in Section 2.5, the anchoring of MNPs onto the polymeric support became noticeable with a change of color from white to brown. Additionally, SEM micrographs of 
materials were performed (Fig. 2). As it can be seen, MNPs are successfully attached to the polymeric surface.
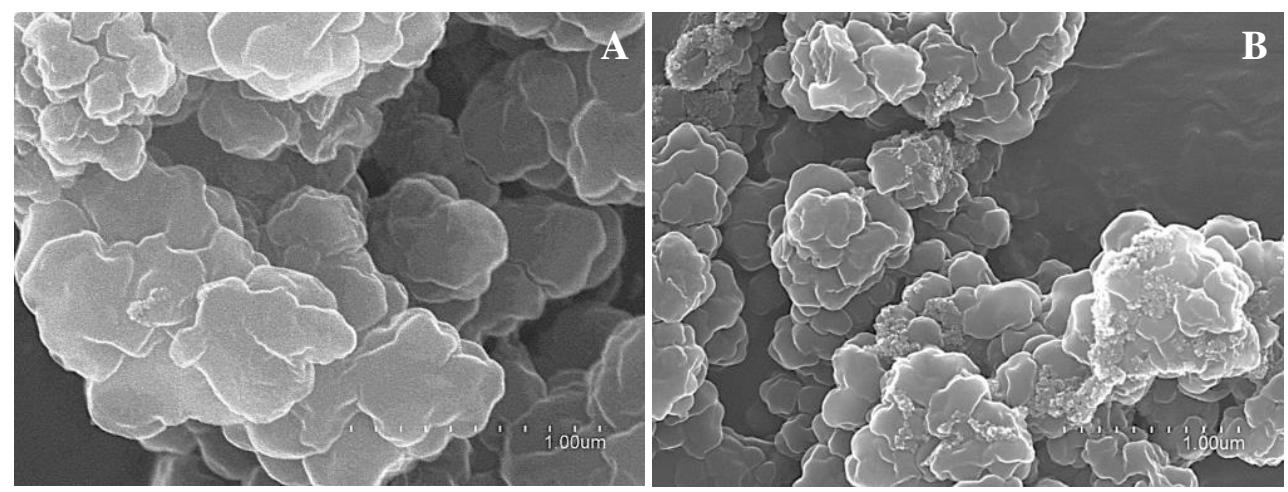

Fig. 2. SEM micrographs of (GMA-co-EDMA) material without MNPs (A) and modified with MNPs (B).

On the other hand, iron content of the MNPs-modified material was evaluated using both UV-Vis and ICP-MS. Thus, UV-Vis spectroscopy analysis showed the presence of (1.69 \pm $0.08) \mathrm{wt} \% \mathrm{Fe}$, being ICP-MS analysis $((1.59 \pm 0.03) \mathrm{wt} \% \mathrm{Fe})$ consistent with this value. The application of a statistical $t$-test to these data showed no significant differences at the $95 \%$ confidence level. In this work, iron percentage is in the range content reported for other organic polymers modified with iron oxide nanoparticles (1\% [19] and 3.7\% [26]), where the measurements were performed by energy dispersive X-ray analysis (EDAX). Additionally, the surface area of the resulting modified polymer was evaluated. The analysis of this material showed a significantly higher surface area $\left(15.17 \mathrm{~m}^{2} \mathrm{~g}^{-1}\right)$ compared to that of the unmodified polymer $\left(6.14 \mathrm{~m}^{2} \mathrm{~g}^{-1}\right)$. This increase in surface area results in an additional benefit to the retention of target analytes.

\subsection{Selection of SPE conditions with the MNPs-modified material}

The selection of an appropriate solvent is of major concern to optimize a SPE process. Since our aim was to use the MNPs-modified material to extract PLs from lipid extracts, several solvents were selected to load the sample solution. Initially, a $\mathrm{CHCl}_{3}: \mathrm{MeOH}$ mixture $(2: 1, \mathrm{v} / \mathrm{v})$ was chosen, since this is the solvent commonly used to extract lipids [27-30]. However, when this solvent mixture was employed, $55 \%$ of the loaded amount of PC (250 $\mu \mathrm{g}$ of PC) was lost. For this reason, organic solvent mixtures with greater hydrophobic character, which are able to dissolve fat-based matrices containing PLs, were considered [31]. Thus, the same amount of PC (250 $\mu \mathrm{g}$ of PC) dissolved in hexane-EtOH $(98: 2, \mathrm{v} / \mathrm{v})$ was percolated through the SPE cartridge. 
PC content found in the percolated fraction was accounted for only $3 \%$ of initial PC. Several studies [15,32] have described that the adsorption of PLs on the surface of MNPs could be explained taking into account two driving forces: i) an entropy increase due to displacement of solvent molecules around NPs by the PL molecules or ii) charge-dipole attraction between the charged NPs and the P-N dipole of the PL headgroups. The results suggested that when more polar solvents were employed, MNPs as well as PC were preferentially swollen, which had a detrimental effect on their interaction, and consequently, in PC adsorption.

Once established the loading solvent, an appropriate washing step was introduced. Since the hydrophobic character of hexane can be suitable to remove matrix nonpolar components ( $e$. g. triglycerides) without jeopardizing PLs adsorption onto the MNPs, it was initially selected as washing solvent. Next, retained PC was desorbed with $\mathrm{CHCl}_{3}: \mathrm{MeOH}(2: 1, \mathrm{v} / \mathrm{v})$, since the polarity of the mixture was expected to compete with PC-MNPs interaction, thus ensuring high extraction efficiency of the target analytes. Following the described procedure, no loss of PC was observed in the washing step, and PC recovery in the elution fraction reached values above $95 \%$.

Under the selected conditions, solutions of SM and PE $\left(500 \mu \mathrm{L}, 500 \mu \mathrm{g} \mathrm{mL} \mathrm{m}^{-1}\right.$ each $)$ were percolated through the SPE cartridge. Recovery values for these PLs were 92\% and 93\%, respectively. The SPE protocol was also applied to a GMA-co-EDMA cartridge (without MNPs), and recovery values around $40 \%$ for all investigated PLs were obtained. These results confirmed that the attached MNPs onto the GMA-based polymer played an important role in the adsorption of these compounds.

\subsection{Analytical features of the MNPs-SPE cartridge}

First, increased amounts of PC standard were percolated through the MNPs-cartridge following the extraction protocol described in Section 2.6. It was intended to establish the maximum loading capacity of the MNPs-cartridge for PC adsorption. Fig. 3 shows that after loading $1000 \mu \mathrm{g}$ of PC (6.7 $\mu \mathrm{g}$ of PC mg ${ }^{-1}$ of sorbent), adsorption on the MNPs-cartridge diminishes substantially ( $c a$. 60\%). Thus, the adsorption capacity of the MNPs-cartridge was established in $5.7 \mu \mathrm{g}$ of PC per mg of polymer.

Then, the breakthrough volume, defined as the sample volume that can be loaded on the sorbent bed without loss of the analytes, was evaluated. Thus, variable volumes of PC standard solutions $(0.5-5.0 \mathrm{~mL})$, keeping constant the total amount of PC $\left(1.7 \mu \mathrm{g} \mathrm{PC} \mathrm{mg}{ }^{-1}\right.$ of sorbent), were percolated through the MNPs-cartridge and the SPE protocol was applied. In the range assayed, no significant losses of PC $(<4 \%)$ were observed (spectrophotometrically quantified [24]). 


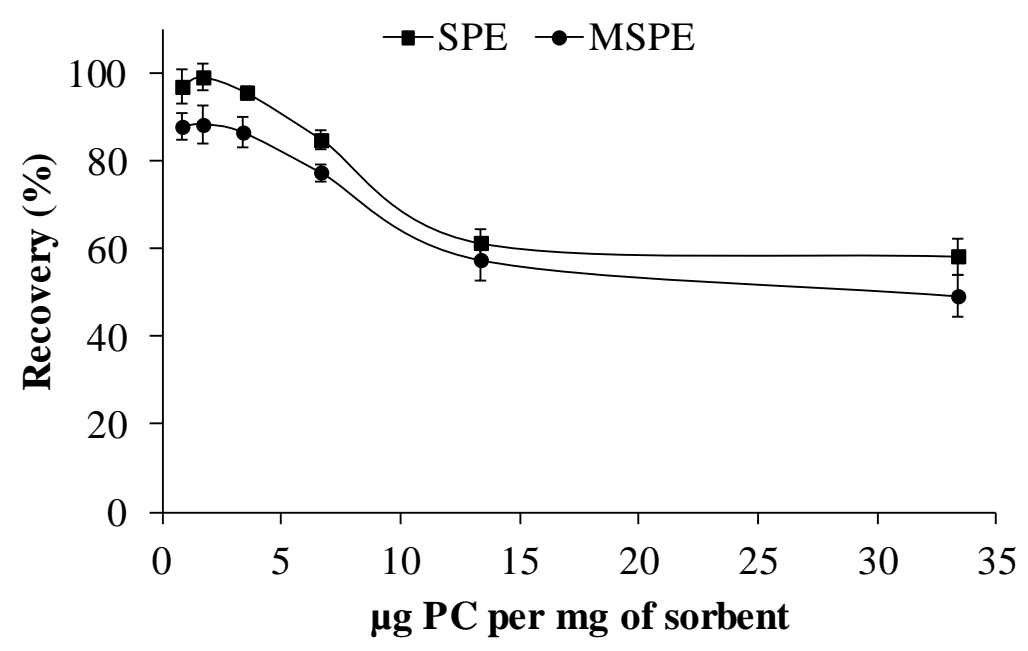

Fig. 3. Adsorption capacity at increasing PC amounts of the MNPs material in SPE and MSPE modes.

The reusability of the SPE columns was evaluated (250 $\mu \mathrm{g}$ of PC) using the proposed procedure. A single MNPs-SPE column was repeatedly used $(n=10)$ using the regeneration protocol described in Section 2.6.An excellent performance with recoveries higher than $88 \%$ for PC was observed.

\subsection{Extraction of PC in the MSPE mode}

The MSPE protocol using the MNPs-modified material under application of an external magnetic field was developed (see Section 2.7). For this purpose, several parameters previously studied in the conventional SPE procedure were adapted to MSPE. In order to compare the performance in both modalities, the same amount of sorbent $(150 \mathrm{mg})$ was employed. First, adsorption capacity was evaluated with $5 \mathrm{~mL}$ of PC standard solutions (125 - $5000 \mu \mathrm{g}$ of PC). Recovery of PC followed the same trend found for SPE, although the values found were lower (see Fig. 3). Since sonication has been proved to increase the efficiency of desorption of analytes from a solid sorbent $[33,34]$, the elution step was assisted by sonication at two levels: 250 and $2000 \mu \mathrm{g}$ of PC. In this study, however, sonication improved only slightly the recoveries (1-5\%) and therefore, desorption assisted with sonication was not further considered. Thus, the loading capacity of the MNPs-modified material in MSPE was established in $3.3 \mu \mathrm{g}$ of PC mg of polymer.

Then, reusability of the MNPs-modified material used in MSPE was evaluated (250 $\mu \mathrm{g}$ of PC). For this purpose, the regeneration and conditioning processes carried out for the SPE cartridges were adopted. In MSPE, extraction capacity of the MNPs material in MSPE was reduced in its first use up to 53\%, so that the MNPs modified polymer should not be used more than 1 time in this sorption modality. The reason for this limited reusability may be attributed to 
the following reasons. On the one hand, a loss of mass of the MNPs-modified material after the regeneration process, since the sorbent was initially weighed and washed once using different solvents $\left(\mathrm{CHCl}_{3}, \mathrm{MeOH}\right.$ and hexane-EtOH $\left.(98: 2, \mathrm{v} / \mathrm{v})\right)$. On the other hand, the bare MNPs can undergo a loss in their magnetic properties upon direct exposing to certain surrounding environment (e.g. solvents), which produces a destabilization effect caused by electrostatic, hydrophobic or hydrogen bond interactions [35]. In fact, the magnetic field has a greater effect on the solvents with stronger hydrogen bond and becomes an obstacle to the diffusion of solute molecules [36].

As stated before, PC recoveries obtained via conventional SPE and via MSPE were comparable, although slightly better results were found in the first approach. However, regeneration process seems to be determining in MSPE, and to continue with this approach would imply the use of large amounts of sorbent. On the other hand, the SPE protocol simplifies largely the handling of more samples simultaneously, which would accelerate the clean-up process of fat extracts. For this reason, the SPE approach was selected for further studies.

\subsection{Extraction and analysis of PLs from lipid extracts of milk}

The applicability of the MNPs-modified material was evaluated percolating through the SPE-cartridge human milk fat extracts. Thus, the protocol described in Section 2.6 was followed, and the analysis of PC, PE and SM was accomplished by HILIC-ELSD. To evaluate quantitatively these three PLs, external calibration curves for each PL $\left(20-400 \mu \mathrm{g} \mathrm{mL}^{-1}\right.$ in $\left.\mathrm{CHCl}_{3}: \mathrm{MeOH}(2: 1, \mathrm{v} / \mathrm{v})\right)$ were constructed. Peak area $v s$ mass of lipid values were fitted both to linear and power models (Table S1). Since linear regression gave reasonable coefficients of determination for all analytes, in the adopted concentration range, it was selected for quantitation studies. Limits of detection (LODs) and quantitation (LOQs) were determined from 3 and 10 signal-to-noise ratio, respectively. As shown in Table S1, LODs are comprised between 1.4 and $3.5 \mu \mathrm{g} \mathrm{mL}{ }^{-1}$, and LOQs between 4.8 and $11.7 \mu \mathrm{g} \mathrm{mL}^{-1}$. To evaluate precision of the HILIC-ELSD method in terms of retention times and peak areas, a multi-component standard solution $\left(100 \mu \mathrm{g} \cdot \mathrm{mL}^{-1}\right)$ was injected 3 times per day for 3 days. For all the analytes considered, relative standard deviations (RSD) lower than $2.5 \%$ were obtained.

Fig. S3 shows the chromatograms of a human milk fat extract before and after applying the SPE protocol with the MNPs-based sorbent. A significant decrease of nonpolar lipids and a large enhancement of signals corresponding to PLs are observed in the elution fraction (continuous line) with respect to the untreated fat extract (dashed line). This fact confirms the suitability of the MNPs-modified material to be used as sorbent in SPE for clean-up and preconcentration purposes, allowing further characterization of target analytes (PLs). On the other, considering the complexity of milk matrices and the unavailability of an analyte-free milk sample, a recovery study using the standard addition method was conducted. Thus, a human 
milk sample $(5 \mathrm{~mL})$ was fortified at four concentration levels (between 10 and $50 \mu \mathrm{g} \mathrm{mL}^{-1}$ ) and these spiked samples were analyzed after applying the MNPs-SPE procedure. Recovery values of the spiked PLs were comprised between 83 and $90 \%$, which suggests that their extraction was unaffected by the matrix.

Then, reusability of the MNPs-SPE cartridges for fat extracts was also evaluated. For this purpose, fat extracts were dissolved in $5 \mathrm{~mL}$ of hexane-EtOH $(98: 2, \mathrm{v} / \mathrm{v})$ and the adsorption-elution cycle was repeated 6 times using the same MNPs-SPE cartridge. To evaluate retention, the total content of PLs was estimated before and after applying the SPE protocol by the Stewart assay [24]. Fig. S4 shows recovery values of PLs from human milk fat extracts as a function of increasing number of reuses. It can be seen that the sorbent demonstrates an appropriate performance for fat analysis at least for three consecutive uses (RSD 8.0\%).

Finally, the content of PLs of several human milk samples in different lactation stages (colostrum, transitional, and mature milk) was determined using the described protocol. As shown in Table S2, some variability in the PL levels can be observed among the analyzed human milk samples. In all cases, the amount of PLs is in good agreement with the data reported for these compounds in human milk samples [29,37]. Additionally, a decrease in PL content as lactation progresses is observed, which is in accordance with the findings previously reported $[3,38]$.

The developed method was also compared with other extraction methods [5,6,39-44], being SPE the commonly used technique [5,6,39-41]. Regarding recoveries, our values were similar to those reported in literature $[5,6,43,39,41]$, which demonstrates the suitability of the procedure. Concerning LODs and LOQs, our SPE protocol gave comparable values to those reported using ELSD [6], but higher than those obtained using more sophisticated techniques, such as MS [43,39]. In addition, the amount of sorbent in the SPE cartridges for PLs analysis ranges from $25 \mathrm{mg}$ for home-made cartridges [41] to $1000 \mathrm{~g}$ for commercial cartridges [5,45]. In this sense, the amount employed in this work $(150 \mathrm{mg})$ allows us to prepare several SPE cartridges ( $c a$. 15) from the bulk MNPs-modified material. Additionally, since some sorbents reported [45,46] might not be affordable for all laboratories, our home-made cartridges represent undoubtedly a potential alternative.

\section{Conclusions}

In this work, a polymeric material was successfully functionalized with MNPs and then used as sorbent for the extraction of PLs from human milk samples. Subsequently, the extracted PLs were separated and quantified by HILIC-ELSD. To our knowledge, the present study is the first work in using a MNPs-modified material as sorbent for the extraction of PLs from this complex matrix. The use of bare $\mathrm{Fe}_{3} \mathrm{O}_{4} \mathrm{NPs}$ attached to the surface of the polymeric support constitutes a favorable platform for the selective interaction of phosphorous-compounds, such as 
PLs. Although the magnetic functionalized material could be used both in SPE and MSPE, the SPE procedure presented some advantages (e.g. slightly higher recoveries, better reusability and a large high-throughput) over the MSPE methodology.

The performance of the MNPs-SPE cartridge was demonstrated by its application to human milk fat samples, where an effective clean-up of sample (particularly to remove neutral lipids) and preconcentration of PLs was accomplished. Following this methodology, it is possible an accurate determination of PLs by HILIC-ELSD while the structural integrity of the HPLC column is guaranteed. Thus, the proposed methodology represents a promising alternative for the extraction of PLs from biological samples.

\section{Acknowledgments}

Project CTQ2014-52765-R (MINECO of Spain and FEDER) and PROMETEO/2016/145 (Generalitat Valenciana). I. T-D thanks the MINECO for an FPU grant for PhD studies.

\section{References}

[1] R.G. Jensen, Lipids in human milk, Lipids. 34 (1999) 1243-1271. doi:10.1007/s11745999-0477-2.

[2] L. Wang, Y. Shimizu, S. Kaneko, S. Hanaka, T. Abe, H. Shimasaki, H. Hisaki, H. Nakajima, Comparison of the fatty acid composition of total lipids and phospholipids in breast milk from Japanese women, Pediatr. Int. 42 (2000) 14-20. doi:10.1046/j.1442200X.2000.01169.x.

[3] J. Bitman, L. Wood, M. Hamosh, P. Hamosh, N.R. Mehta, Comparison of the lipid composition of breast milk from mothers of term and preterm infants, Am. J. Clin. Nutr. 38 (1983) 300-312. http://www.ncbi.nlm.nih.gov/pubmed/6881084.

[4] R.G. Jensen, The lipids in human milk, Prog. Lipid Res. 35 (1996) 53-92. doi:10.1016/0163-7827(95)00010-0.

[5] A. Avalli, G. Contarini, Determination of phospholipids in dairy products by SPE/HPLC/ELSD, J. Chromatogr. A. $1071 \quad$ (2005) 185-190. doi:10.1016/j.chroma.2005.01.072.

[6] P. Donato, F. Cacciola, F. Cichello, M. Russo, P. Dugo, L. Mondello, Determination of phospholipids in milk samples by means of hydrophilic interaction liquid chromatography coupled to evaporative light scattering and mass spectrometry detection, J. Chromatogr. A. 1218 (2011) 6476-6482. doi:10.1016/j.chroma.2011.07.036.

[7] M. Schwalbe-Herrmann, J. Willmann, D. Leibfritz, Separation of phospholipid classes by hydrophilic interaction chromatography detected by electrospray ionization mass 
spectrometry., J. Chromatogr. A. $1217 \quad$ (2010) 5179-5183. doi:10.1016/j.chroma.2010.05.014.

[8] J.G. Parsons, S. Patton, Two-dimensional thin-layer chromatography of polar lipids from milk and mammary tissue, J. Lipid Res. 8 (1967) 696-698.

[9] F. Sánchez-Juanes, J.M. Alonso, L. Zancada, P. Hueso, Distribution and fatty acid content of phospholipids from bovine milk and bovine milk fat globule membranes, Int. Dairy J. 19 (2009) 273-278. doi:10.1016/j.idairyj.2008.11.006.

[10] R.J. Maxwell, D. Mondimore, J. Tobias, Rapid method for the quantitative extraction and simultaneous class separation of milk lipids, J. Dairy Sci. 69 (1986) 321-325. doi:10.3168/jds.S0022-0302(86)80408-8.

[11] M. Wierucka, M. Biziuk, Application of magnetic nanoparticles for magnetic solidphase extraction in preparing biological, environmental and food samples, Trends Anal. Chem. 59 (2014) 50-58. doi:10.1016/j.trac.2014.04.007.

[12] I.S. Ibarra, J.A. Rodriguez, C.A. Galán-Vidal, A. Cepeda, J.M. Miranda, Magnetic solid phase extraction applied to food analysis, J. Chem. (2015) 13. doi:10.1155/2015/919414.

[13] A.H. Lu, E.L. Salabas, F. Schüth, Magnetic nanoparticles: Synthesis, protection, functionalization, and application, Angew. Chemie - Int. Ed. 46 (2007) 1222-1244. doi:10.1002/anie.200602866.

[14] L. Weng, W.H. Van Riemsdijk, T. Hiemstra, Factors controlling phosphate interaction with iron oxides, J. Environ. Qual. 41 (2012) 628-635. doi:10.2134/jeq2011.0250.

[15] S. Zhang, H. Niu, Y. Zhang, J. Liu, Y. Shi, X. Zhang, Y. Cai, Biocompatible phosphatidylcholine bilayer coated on magnetic nanoparticles and their application in the extraction of several polycyclic aromatic hydrocarbons from environmental water and milk samples, J. Chromatogr. A. 1238 (2012) 38-45. doi:10.1016/j.chroma.2012.03.056.

[16] T. Nema, E.C.Y. Chan, P.C. Ho, Applications of monolithic materials for sample preparation, J. Pharm. Biomed. Anal. 87 (2014) 130-141. doi:10.1016/j.jpba.2013.05.036.

[17] M. Vergara-Barberán, M.J. Lerma-García, E.F. Simó-Alfonso, J.M. Herrero-Martínez, Solid-phase extraction based on ground methacrylate monolith modified with gold nanoparticles for isolation of proteins, Anal. Chim. Acta. 917 (2016) 37-43. doi:10.1016/j.aca.2016.02.043.

[18] I.D. Vukoje, E.S. Džunuzović, D.R. Lončarević, S. Dimitrijević, S.P. Ahrenkiel, J.M. Nedeljković, Synthesis, characterization, and antimicrobial activity of silver nanoparticles on poly(GMA-co-EGDMA) polymer support, Polym. Compos. (2015) 19. doi:10.1002/pc.23684. 
[19] J. Krenkova, F. Foret, Iron oxide nanoparticle coating of organic polymer-based monolithic columns for phosphopeptide enrichment, J. Sep. Sci. 34 (2011) 2106-2112. doi:10.1002/jssc.201100256.

[20] S. Meseguer-Lloret, S. Torres-Cartas, M. Catalá-Icardo, E.F. Simó-Alfonso, J.M. Herrero-Martínez, Extraction and preconcentration of organophosphorus pesticides in water by using a polymethacrylate-based sorbent modified with magnetic nanoparticles, Anal. Bioanal. Chem. 409 (2017) 3561-3571. doi:10.1007/s00216-017-0294-x.

[21] C. Yang, G. Wang, Z. Lu, J. Sun, J. Zhuang, W. Yang, Effect of ultrasonic treatment on dispersibility of $\mathrm{Fe}_{3} \mathrm{O}_{4}$ nanoparticles and synthesis of multi-core $\mathrm{Fe}_{3} \mathrm{O}_{4} / \mathrm{SiO}_{2}$ core/shell nanoparticles, J. Mater. Chem. 15 (2005) 4252-4257. doi:10.1039/b505018a.

[22] E.J. Carrasco-Correa, G. Ramis-Ramos, J.M. Herrero-Martínez, Methacrylate monolithic columns functionalized with epinephrine for capillary electrochromatography applications, J. Chromatogr. A. 1298 (2013) 61-67. doi:10.1016/j.chroma.2013.05.013.

[23] P. Serra-Mora, Y. Moliner-Martínez, R. Herráez-Hernández, J. Verdú-Andrés, P. Campíns-Falcó, Simplifying iron determination with o-phenanthroline in food ashes using 2-nitrophenol as an acid-base indicator, Food Anal. Methods. 9 (2016) 1150-1154. doi:10.1007/s12161-015-0294-4.

[24] J.C.M. Stewart, Colorimetric determination of phospholipids with ammonium ferrothiocyanate, Anal. Biochem. 104 (1980) 10-14. doi:10.1016/0003-2697(80)902699.

[25] J. Folch, M. Lees, G.H. Sloane Stantley, A simple method for the isolation and purification of total lipides from animal tissues, J. Biol. Chem. 266 (1957) 497-509.

[26] J. Krenkova, F. Foret, Nanoparticle-modified monolithic pipette tips for phosphopeptide enrichment, Anal. Bioanal. Chem. 405 (2013) 2175-2183. doi:10.1007/s00216-0126358-z.

[27] C. Lopez, V. Briard-Bion, O. Menard, F. Rousseau, P. Pradel, J.-M. Besle, Phospholipid, sphingolipid, and fatty acid compositions of the milk fat globule membrane are modified by diet, J. Agric. Food Chem. 56 (2008) 5226-5236. doi:10.1021/jf7036104.

[28] L.M. Rodríguez-Alcalá, J. Fontecha, Major lipid classes separation of buttermilk, and cows, goats and ewes milk by high performance liquid chromatography with an evaporative light scattering detector focused on the phospholipid fraction., J. Chromatogr. A. 1217 (2010) 3063-3066. doi:10.1016/j.chroma.2010.02.073.

[29] F. Giuffrida, C. Cruz-Hernandez, B. Flück, I. Tavazzi, S.K. Thakkar, F. Destaillats, M. Braun, Quantification of phospholipids classes in human milk, Lipids. 48 (2013) 10511058. doi:10.1007/s11745-013-3825-z.

[30] T.P.L. Ferraz, M.C. Fiúza, M.L.A. Dos Santos, L. Pontes De Carvalho, N.M. Soares, Comparison of six methods for the extraction of lipids from serum in terms of 
effectiveness and protein preservation, J. Biochem. Biophys. Methods. 58 (2004) 187193. doi:10.1016/j.jbbm.2003.10.008.

[31] K.M. Marakulina, R. V. Kramor, Y.K. Lukanina, M. V. Kozlov, L.N. Shishkina, Application of UV- and IR-Spectroscopy to analyze the formation of complexes between sphingomyelin and phenolic antioxidants, Moscow Univ. Chem. Bull. 67 (2012) 185191. doi:10.3103/S0027131412040098.

[32] B. Yuan, L.L. Xing, Y.D. Zhang, Y. Lu, Z.H. Mai, M. Li, Self-assembly of highly oriented lamellar nanoparticle-phospholipid nanocomposites on solid surfaces, J. Am. Chem. Soc. 129 (2007) 11332-11333. doi:10.1021/ja074235n.

[33] R.S. Davidson, a. Safdar, J.D. Spencer, B. Robinson, Applications of ultrasound to organic chemistry, Ultrasonics. 25 (1987) 35-39. doi:10.1016/0041-624X(87)90009-6.

[34] R. Sitko, B. Gliwinska, B. Zawisza, B. Feist, Ultrasound-assisted solid-phase extraction using multiwalled carbon nanotubes for determination of cadmium by flame atomic absorption spectrometry, J. Anal. At. Spectrom. 28 (2013) 405-410. doi:10.1039/C2JA30328K.

[35] D.P. Joshi, G. Pant, N. Arora, S. Nainwal, Effect of solvents on morphology, magnetic and dielectric properties of $\left(\alpha-\mathrm{Fe}_{2} \mathrm{O}_{3} @ \mathrm{SiO}_{2}\right)$ core-shell nanoparticles, Heliyon. 3 (2017) 1-16. doi:10.1016/j.heliyon.2017.e00253.

[36] F. Moosavi, M. Gholizadeh, Magnetic effects on the solvent properties investigated by molecular dynamics simulation, J. Magn. Magn. Mater. 354 (2014) 239-247. doi:10.1016/j.jmmm.2013.11.012.

[37] G. Contarini, M. Povolo, Phospholipids in milk fat: composition, biological and technological significance, and analytical strategies, Int. J. Mol. Sci. 14 (2013) 28082831. doi:10.3390/ijms14022808.

[38] H. Shoji, T. Shimizu, N. Kaneko, K. Shinohara, S. Shiga, M. Saito, K. Oshida, T. Shimizu, M. Takase, Y. Yamashiro, Comparison of the phospholipid classes in human milk in Japanese mothers of term and preterm infants, Acta Paediatr. 95 (2006) 9961000. doi:10.1080/08035250600660933.

[39] Q. Shen, H.-Y. Cheung, $\mathrm{TiO}_{2} / \mathrm{SiO}_{2}$ core-shell composite-based sample preparation method for selective extraction of phospholipids from shrimp waste followed by hydrophilic interaction chromatography coupled with quadrupole time-of-flight/mass spectrometry analysis, J. Agric. Food Chem. 62 (2014) 8944-8951. doi:10.1021/jf503040p.

[40] C. Ferreiro-Vera, F. Priego-Capote, M.D. Luque de Castro, Comparison of sample preparation approaches for phospholipids profiling in human serum by liquid chromatography-tandem mass spectrometry, J. Chromatogr. A. 1240 (2012) 21-28. doi:10.1016/j.chroma.2012.03.074. 
[41] I. Ten-Doménech, H. Martínez-Pérez-Cejuela, M.J. Lerma-García, E.F. Simó-Alfonso, J.M. Herrero-Martínez, Molecularly imprinted polymers for selective solid-phase extraction of phospholipids from human milk samples, Microchim. Acta. 184 (2017) 3389-3397. doi:10.1007/s00604-017-2345-6.

[42] C.D. Calvano, O.N. Jensen, C.G. Zambonin, Selective extraction of phospholipids from dairy products by micro-solid phase extraction based on titanium dioxide microcolumns followed by MALDI-TOF-MS analysis, Anal. Bioanal. Chem. 394 (2009) 1453-1461. doi:10.1007/s00216-009-2812-y.

[43] S.K. Kailasa, H.F. Wu, Surface modified $\mathrm{BaTiO}_{3}$ nanoparticles as the matrix for phospholipids and as extracting probes for LLME of hydrophobic proteins in Escherichia coli by MALDI-MS, Talanta. 114 (2013) 283-290. doi:10.1016/j.talanta.2013.05.032.

[44] C. Pegoraro, D. Silvestri, G. Ciardelli, C. Cristallini, N. Barbani, Molecularly imprinted poly(ethylene-co-vinyl alcohol) membranes for the specific recognition of phospholipids, Biosens. $\quad$ Bioelectron. $24 \quad$ (2008) 748-755. doi:10.1016/j.bios.2008.06.050.

[45] Sigma-Aldrich, Supelclean $^{\mathrm{TM}}$ LC-Si SPE tube, (n.d.). http://www.sigmaaldrich.com/catalog/product/supelco/57051?lang=es\&region=ES (accessed April 6, 2017).

[46] Sigma-Aldrich, HybridSPE®-Phospholipid, http://www.sigmaaldrich.com/catalog/product/supelco/55261u?lang=es\&region=ES (accessed May 4, 2017). 


\section{SUPPLEMENTARY MATERIAL}

\section{Experimental}

\section{Chromatographic and detection conditions}

Separation was carried out with a Kinetex ${ }^{\mathrm{TM}}$ HILIC $100 \AA$ A column $(150 \mathrm{~mm} \times 4.6 \mathrm{~mm}$, $2.6 \mu \mathrm{m}$; Phenomenex, Torrance, CA, USA). Mobile phases consisted of ACN-ammonium formate $100 \mathrm{mM}(\mathrm{A}, 97: 3, \mathrm{v} / \mathrm{v})$ and water-ammonium formate $100 \mathrm{mM}(\mathrm{B}, 97: 3, \mathrm{v} / \mathrm{v})$. The chromatographic separation was carried out using the following gradient: $0-3.5 \mathrm{~min}, 100 \% \mathrm{~A}$; in $11 \mathrm{~min}, 15 \% \mathrm{~B}$ is reached and kept during $10 \mathrm{~min}$. Column temperature, $25^{\circ} \mathrm{C}$; flow rate, $1.0 \mathrm{~mL}$ $\min ^{-1}$ and injection volume, $20 \mu \mathrm{L}$. The ELSD parameters were: evaporation and nebulization temperature, 80 and $40^{\circ} \mathrm{C}$, respectively; gas flow rate, 1.4 Standard Litres per Minute (SLM); gain factor, 1 .

\section{Preparation of MNPs}

$180 \mathrm{~mL}$ of $62 \mathrm{mM} \mathrm{Fe}^{3+}$ and $31 \mathrm{mM} \mathrm{Fe}^{2+}$ aqueous solutions were placed into a two-neck round-bottom flask with a glass condenser under nitrogen atmosphere. The solution was heated until reaching $50^{\circ} \mathrm{C}$, at which point $12.5 \mathrm{~mL}$ of concentrated ammonia were added under vigorous stirring. After $30 \mathrm{~min}$, the temperature was raised to $90^{\circ} \mathrm{C}$ and the mixture was kept for another $30 \mathrm{~min}$ at this temperature. The MNPs were collected on the flask wall with a neodymium magnet, washed with water and $\mathrm{EtOH}$ several times to remove excess of reagents and dried at $60^{\circ} \mathrm{C}$ for $30 \mathrm{~min}$. The average diameter of the synthesized $\mathrm{Fe}_{3} \mathrm{O}_{4}$ nanoparticles, measured by TEM, was $c a .12 \mathrm{~nm}$ (see Fig. S1).

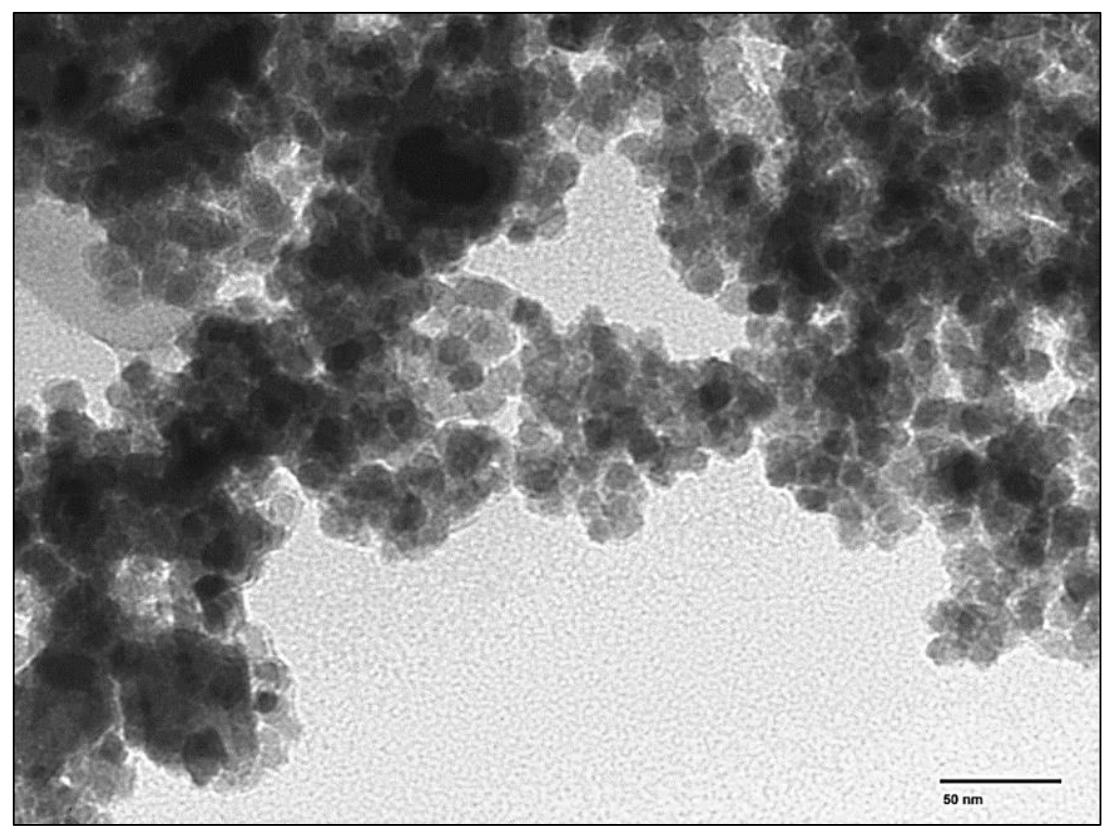

Fig. S1. TEM image of synthesized $\mathrm{Fe}_{3} \mathrm{O}_{4}$ nanoparticles. 
Fe content determination by $U V$-Vis and ICP-MS

For UV-Vis, $25 \mathrm{mg}$ of the dried methacrylate material modified with $\mathrm{Fe}_{3} \mathrm{O}_{4}$ was calcined at $550^{\circ} \mathrm{C}$ for $2.5 \mathrm{~h}$. Then, the ashes (which contained the iron material) were treated with $2 \mathrm{~mL} \mathrm{HCl}$-water $(1: 1, \mathrm{v} / \mathrm{v})$ for $60 \mathrm{~min}$ at $60^{\circ} \mathrm{C}$ under stirring. The resulting solution was subjected to the colorimetric determination of $\mathrm{Fe}$ based on the formation of the red $o$ phenantroline complex. A stock solution of $200 \mu \mathrm{g} \mathrm{mL} \mathrm{m}^{-1}$ of $\mathrm{Fe}$ (II) was prepared from Mohr's salt and a calibration curve $\left(0.8-4 \mu \mathrm{g} \mathrm{mL}^{-1}\right)$ was constructed. Similarly, ashes from calcination of $25 \mathrm{mg}$ of the $\mathrm{Fe}_{3} \mathrm{O}_{4}$ modified material were digested with nitric acid, and the resulting solution was subjected to ICP-MS analysis.

\section{Fat extraction}

$1.5 \mathrm{~mL}$ of milk were dispersed in dichloromethane: $\mathrm{MeOH}(2: 1, \mathrm{v} / \mathrm{v})$ and the mixture was shaken for $15 \mathrm{~min}$ and later centrifuged at $6000 \mathrm{rpm}(3904 \mathrm{~g}$ ) for $8 \mathrm{~min}$. Then, the aqueous phase was removed and the lower dichloromethane phase, containing lipids, was washed with a salt solution and evaporated under vacuum.

\section{SPE and MSPE protocol}

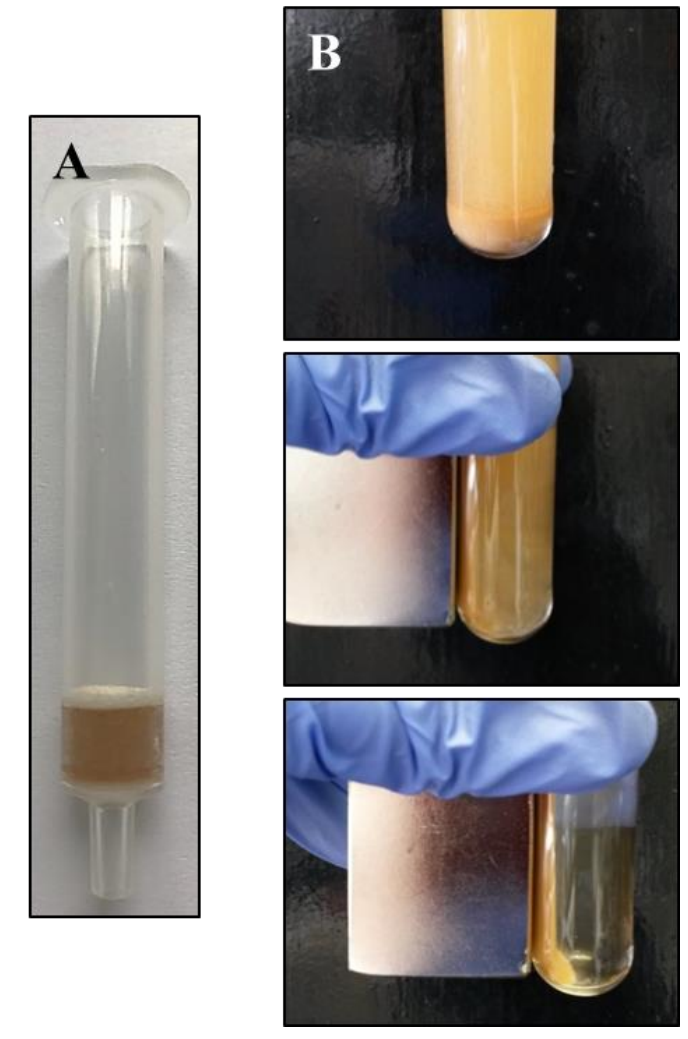

Fig. S2. SPE cartridge with polymeric material modified with MNPs (A); scheme of separation of the MNPs-modified material with a magnet in the extraction steps (B). 
Table S1. Calibration curves (peak area $v s \mu$ injected) and limits of detection (LOD) and quantitation (LOQ) for determination of PLs in HILIC-ELSD.

\begin{tabular}{|c|c|c|c|c|}
\hline PLs & Power & Linear & $\begin{array}{l}\text { LOD } \\
\left(\mu \mathrm{g} \mathrm{mL}^{-1}\right)\end{array}$ & $\begin{array}{l}\text { LOQ }(\mu \mathrm{g} \\
\left.\mathrm{mL}^{-1}\right)\end{array}$ \\
\hline PE & $y=417.1 x^{1.250} ; r^{2}=0.9996$ & $y=931.6 x-1422.7 ; r^{2}=0.9965$ & 1.4 & 4.8 \\
\hline PC & $y=640.1 x^{1.089} ; r^{2}=0.9995$ & $y=794.2 x-145.6 ; r^{2}=0.9983$ & 2.6 & 8.8 \\
\hline SM & $y=471.6 x^{1.045} ; r^{2}=0.9989$ & $y=543.8 x-140.0 ; r^{2}=0.9980$ & 3.5 & 11.7 \\
\hline
\end{tabular}

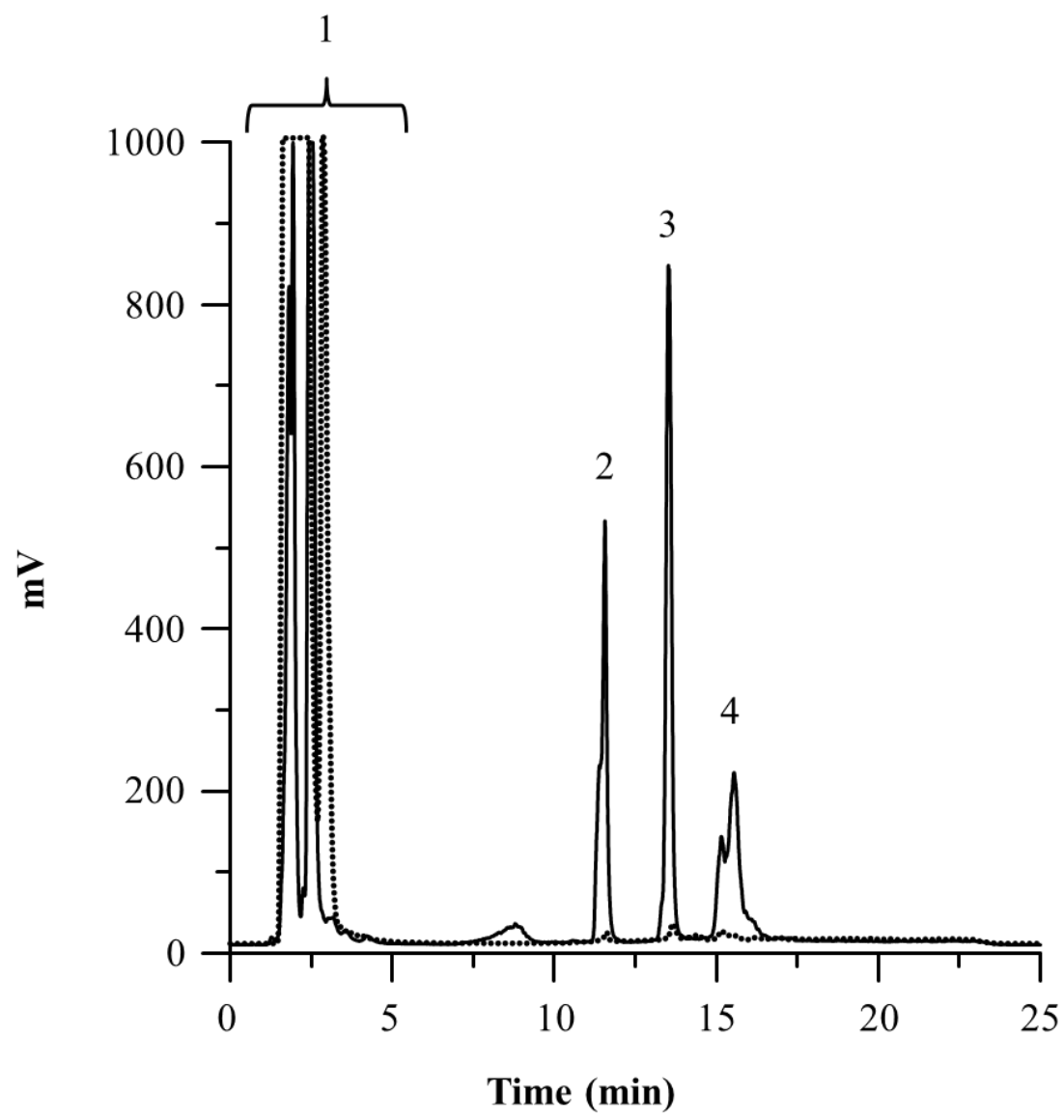

Fig. S3. HILIC-ELSD chromatograms of human milk fat extract obtained without (dashed line) and with (continuous line) MNPs-SPE treatment. For extraction procedure of MNPs-SPE see Section 2.6 and for HILIC-ELSD conditions see above. Peak identification: (1) nonpolar lipids, (2) PE, (3) PC and (4) SM. 


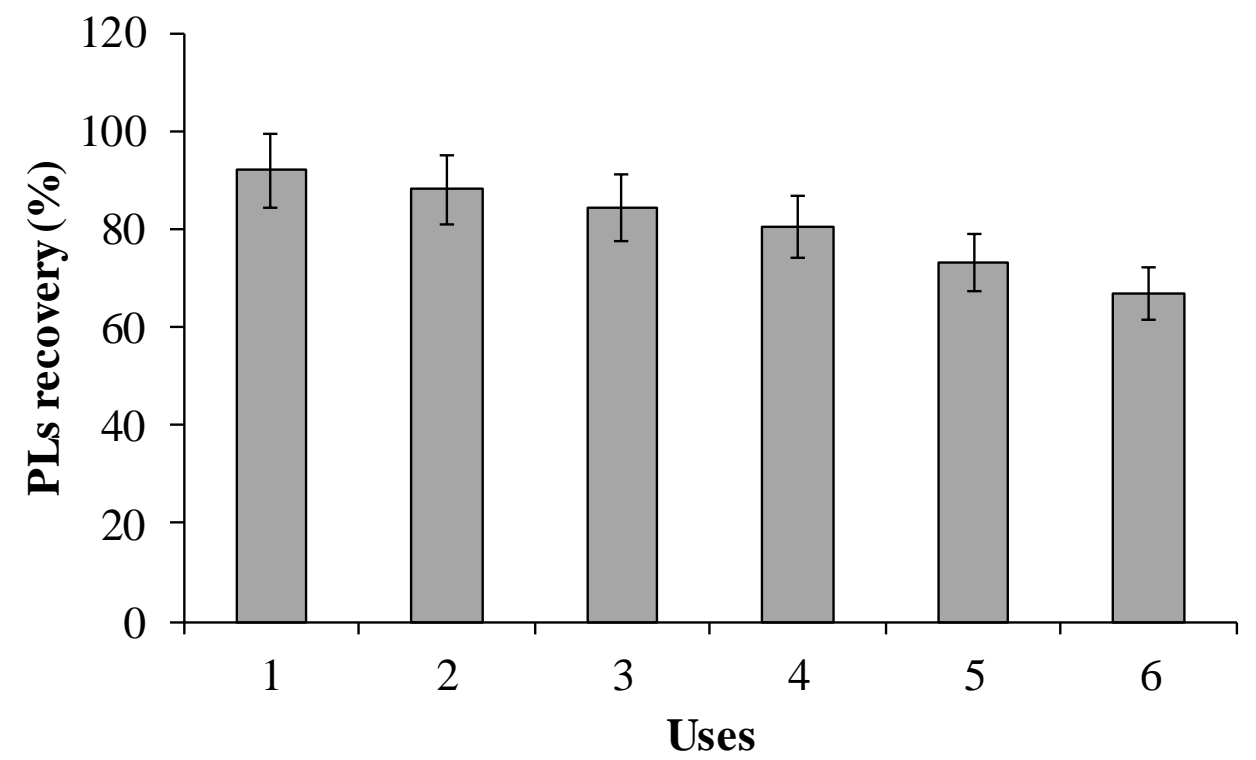

Fig. S4. Recovery values of PLs from human milk fat extracts in SPE as a function of increasing number of reuses. 